\title{
De Novo Assembly and Transcriptome Characterization of Canine Retina Using High-Throughput Sequencing
}

\author{
Bhaskar Reddy, ${ }^{1,2}$ Amrutlal K. Patel, ${ }^{1}$ Krishna M. Singh, ${ }^{1,3}$ Deepak B. Patil, ${ }^{4}$ \\ Pinesh V. Parikh, ${ }^{4}$ Divyesh N. Kelawala, ${ }^{4}$ Prakash G. Koringa, ${ }^{1}$ Vaibhav D. Bhatt, ${ }^{5}$ \\ Mandava V. Rao, ${ }^{2}$ and Chaitanya G. Joshi ${ }^{1}$ \\ ${ }^{1}$ Ome Research Facility, Department of Animal Biotechnology, Anand Agricultural University, Anand, Gujarat 388001, India \\ ${ }^{2}$ Department of Zoology, Genetic Diagnostic Centre, University School of Sciences, Gujarat University, Ahmedabad, \\ Gujarat 380009, India \\ ${ }^{3}$ Datar Genetics Ltd., F-8 D Road, Ambad, Nasik, Maharashtra 422010, India \\ ${ }^{4}$ Department of Veterinary Surgery \& Radiology, College of Veterinary Science \& Animal Husbandry, Anand Agricultural University, \\ Anand, Gujarat 388001, India \\ ${ }^{5}$ Department of Pharmaceutical Science, Saurashtra University, Rajkot, Gujarat 360005, India
}

Correspondence should be addressed to Chaitanya G. Joshi; cgjoshi@rediffmail.com

Received 31 July 2015; Revised 10 November 2015; Accepted 11 November 2015

Academic Editor: Norman A. Doggett

Copyright (C) 2015 Bhaskar Reddy et al. This is an open access article distributed under the Creative Commons Attribution License, which permits unrestricted use, distribution, and reproduction in any medium, provided the original work is properly cited.

We performed transcriptome sequencing of canine retinal tissue by 454 GS-FLX and Ion Torrent PGM platforms. RNA-Seq analysis by CLC Genomics Workbench mapped expression of 10,360 genes. Gene ontology analysis of retinal transcriptome revealed abundance of transcripts known to be involved in vision associated processes. The de novo assembly of the sequences using CAP3 generated 29,683 contigs with mean length of 560.9 and N50 of 619 bases. Further analysis of contigs predicted 3,827 fulllength cDNAs and 29,481 (99\%) open reading frames (ORFs). In addition, 3,782 contigs were assigned to 316 KEGG pathways which included melanogenesis, phototransduction, and retinol metabolism with 33,15 , and 11 contigs, respectively. Among the identified microsatellites, dinucleotide repeats were $68.84 \%$, followed by trinucleotides, tetranucleotides, pentanucleotides, and hexanucleotides in proportions of $25.76,9.40,2.52$, and $0.96 \%$, respectively. This study will serve as a valuable resource for understanding the biology and function of canine retina.

\section{Introduction}

The retina is composed of a neural cell layer and a retinal pigment epithelial cell layer. Two types of photoreceptor cells, rods and cones, in the neural cell layer convert light signals to changes in membrane potential, organized through complex layers of the neural cells and transmitted to the brain through the fibers of the optic nerve $[1,2]$. The retinal pigment epithelium (RPE) plays an important role in supporting the function of the photoreceptor cells and serves as a bloodretina barrier [3]. Photoreceptor cells and the RPE are of interest physiologically as well as pathologically in relation to retinal degeneration [4-7]. Furthermore, photoreceptor cells serve as a model for the investigation of the development and differentiation of neural cells [8] and its normal function is dependent upon each cell type working properly in a coordinated fashion. Multiple disorders, that is, diabetes, agerelated macular degeneration, inherited retinal degeneration (IRD), cancer, and so forth, affect the retina and cause vision loss at all ages [9].

Massively parallel, high-throughput sequencing platforms have provided possibility for genome-wide observations of the transcriptional makeup of retina genes. The transcriptome is the complete set of transcripts in a cell at a specific stage or under given physiological condition [10]. Understanding cell development, physiology, and disease may be improved by genome-wide characterization of the retinal transcriptome [11, 12]. High-throughput mRNA 
sequencing allows simultaneous transcript discovery and abundance estimation [13]. High-throughput sequencing data have a wide dynamic range of transcript expression for quantification and identification of rare transcripts within the constraints of the depth of coverage. With microarray technologies, transcripts can only be detected based on prior knowledge required for probe placement [14]. The size and structure of transcripts can be accurately measured by RNA-Seq, as compared to array hybridization, which does not provide any information on transcript size and splice variation. The microarray based gene expression of canine has been reported for lungs, brain, heart, kidney, liver, lymph node, pancreas, skeletal muscle, and spleen tissues [15]. The characterization of causative mutations for retinal blindness disorders has been of limited success due to poor availability of information on gene expression and underlying molecular mechanisms that trigger degenerative processes. To the best of our knowledge, there are no reports on transcriptome profiling of retinal tissue of dog. Hence, the present study was undertaken with the objective of developing a catalog of genes expressed in canine retina and their functional annotation.

\section{Materials and Methods}

2.1. Tissue Collection. The retinal tissues of both eyes were taken from a female nondescript dog, approximately 4-5 years old, that had an automobile accident and succumbed during the treatment at the Department of Veterinary Surgery \& Radiology, AAU, Anand, Gujarat, India. Tissues were washed with sterile phosphate buffer saline solution, transferred immediately in the "RNA later," and stored in liquid nitrogen for downstream processing.

\section{2. $m R N A$ Extraction, Library Preparation, and Sequencing.} Total RNA was extracted from $100 \mathrm{mg}$ of both tissues using TRIzol (Invitrogen Life Technologies, CA) reagent as per the manufacturer's instructions. DNase treatment was given to remove the DNA contamination. The quantity and quality of RNA were evaluated using NanoDrop1000 spectrophotometer (Thermo Fisher Scientific) as well as Bioanalyzer 2100 (Agilent Technologies, CA). Total mRNA was isolated from total RNA sample using mRNA isolation kit (Roche Diagnostics, Switzerland) as described in the manufacturer's protocols. Total isolated mRNA was again quality checked on Bioanalyzer 2100 using RNA 6000 nano Chip kit (Agilent Technologies, CA). cDNA and library preparations were carried out using kits of 454 GS-FLX sequencing and Ion Torrent mRNA library preparation. Both platforms based cDNA libraries were sequenced on 454 GS-FLX and Ion Torrent PGM sequencers as per the manufacturer's instructions. The brief steps for sequencing are mRNA fragmentation, adapter ligation, cDNA preparation, emulsion PCR based library amplification, and library enrichment.

2.3. Read Mapping and Gene Expression Analysis. The generated reads of both datasets were pooled and subjected to quality screening using PRINSEQ. Reads with less than 60 bases of read length, with mean read quality of less than 20, and with duplicate reads were removed [16]. The quality screened data was processed for the mapping and gene expression analysis. Reads were mapped to canine annotated genome assemblies using CLC Genomics Workbench 4.9 software. To quantify gene expression, the RNA-Seq analysis tool was used as previously described [17] allowing for no more than 2 mismatches per read. The annotated genome was downloaded from NCBI for canine genome build CanFam3.1.

2.4. Functional Annotation of Transcripts. The genes that were expressed with RPKM (reads per kilobase of exon model per million mapped reads) value of $\geq 0.5$ were taken for functional annotation. Genes which were expressed with RPKM < 0.5 were excluded from the functional annotation. The genes (gene ontology) were annotated using Database for Annotation, Visualization and Integrated Discovery (DAVID) Version 6.7 [18] and analyzed for gene enrichment using Functional Annotation Tool. Genes associated with retinal function were retrieved from RetNet retinal information network database (https://sph.uth.edu/retnet/home.htm).

2.5. Sequence Data Processing and De Novo Assembly. The downstream analysis was carried out from total data obtained from 454 GS-FLX and Ion Torrent. The duplicate reads, chimeric reads, minimum length $(<30 \mathrm{bp})$, quality mean read length $(<20)$, and end trimming were performed using PRINSEQ tool [16]. Quality screened data was then processed for assembly. Reads were assembled using contig assembly program-3 (CAP3) [19] with default parameters (overlap similarity score cutoff 90 , overlap percent identity cutoff 90 , mismatch score ( -5$)$, and base quality cutoff 20 ).

2.6. Comparative Annotation of Assembled Contigs. The annotations of contigs were carried out by BLASTx searches of all contigs with reference to the NCBI nonredundant (nr) database using Blast2GO [13] with $e$-value cutoff of $1 * 10^{-6}$. The assembled sequences were subjected to KEGG pathways assignment using the online KEGG [20] Automatic Annotation Server (KAAS) (http://www.genome.jp/kegg/kaas/) Ver. $1.67 \mathrm{x}$ with default parameters. KEGG pathway analyses of contigs were performed on KASS server using Bidirectional Best Hit.

2.7. Full-Length cDNA Prediction. All assembled transcripts (using CAP3) were submitted to in-house local BLASTx against protein sequence database of Canis lupus familiaris with $e$-value cutoff of $10^{-5}$ for identification of full-length cDNA. Prediction of full-length cDNAs was identified using online tool Target Identifier [21] with $e$-value cutoff of $10^{-5}$. The cDNA sequence was recognized as a full-length cDNA only if it has the start codon (ATG) and poly(A) tail codon.

2.8. ORF Identification. The assembled contigs were uploaded to online tool ORFPredictor [22] to identify open reading frames in the assembled contigs with an $e$-value cutoff of $10^{-5}$.

2.9. Identification of SSR/Microsatellites and Repeat Elements. The SSRs were identified from assembled sequences using 
TABLE 1: Number and proportion of reads mapped to reference genome. Mapping statistics were carried out using CLC Genomics with Canis lupus familiaris genome build version CanFam3.1 as a reference (NCBI).

\begin{tabular}{lc}
\hline & Canine retina (expressed genes) \\
\hline Total reads & 800,154 \\
Counted fragments & 226,684 \\
Uniquely mapped & 222,296 \\
Mapped unspecifically & 4,388 \\
Uncounted fragments & 573,470 \\
Number of genes & 10,360 \\
Number of genes in reference & 28,455 \\
\hline
\end{tabular}

SSR Locator [23] with threshold of 6 for di- and 5 for tri-, tetra-, penta-, and hexanucleotide repeats.

\section{Results}

3.1. Sequencing Analysis and Mapping Statistics. RNA sequencing carried out on Ion Torrent yielded a total of 569,066 quality reads with mean read length of $145.79 \mathrm{bp}$ and on 454 GS-FLX yielded a total of 231,088 quality reads with mean read length of $373.19 \mathrm{bp}$. Out of 226,684 counted fragments, 222,296 reads mapped uniquely whereas 4,388 reads mapped nonspecifically with reference genome (Table 1). A total of 10,360 genes were identified out of 28,455 reference genes of canine transcriptome. The annotated reference assembly was downloaded from NCBI Genome browser CanFam3.1 reference genome (ftp://ftp.ncbi.nlm.nih.gov/genomes/Canis_lupus_familiaris/).

Among the reads mapping uniquely to protein coding genes, $34.05 \%$ located within exon reads and $14.63 \%$ on exonexon reads. Nearly $48.00 \%$ located within the introns and $2.75 \%$ in the exon-intron reads (Table 2). The relatively high proportion of reads assigned to introns is not uncommon when the sequencing library preparation includes random priming of the mRNA [24].

3.2. Analysis of Gene Expression Profile of Retina. In our transcriptome study, 10,360 genes were expressed with $R P K M$ values $\geq 0.50$. In order to categorize the genes with different level of expression, genes were categorized based on RPKM values into three groups, that is, high ( $\geq 200$ RPKM), medium ( $\geq 10-200$ RPKM), and low $(\geq 0.4-$ 10 RPKM) expressed genes. There were 36 (0.78\%) highly expressed genes, 1,850 (40.14\%) moderately expressed genes, and $2,723(59.08 \%)$ low expressed genes in the retina.

3.3. Functional Annotation of Retina Expressed Genes (GO Analysis). The functional annotations of genes expressed in retinal tissue were performed using DAVID 6.7 web based annotation tool [18] which provides dynamic, controlled vocabulary and hierarchical relationships for the gene products in three categories: biological process, molecular function, and cellular component. Gene enrichment of GO terms was significant ( $p$ value $<0.01$ ) in biological pathway, molecular function, and cellular component. The biological process was enriched in a total of $28 \mathrm{GO}$ terms, cellular component in $34 \mathrm{GO}$ terms, and molecular function in $27 \mathrm{GO}$ terms.

3.3.1. GO: Biological Process. The enrichment of expressed genes in biological process was observed for 316 genes in 28 GO terms which ranged between 25 and 6 genes. The assignment of GO terms ( $p$ value $<0.01$ ) included intracellular signaling cascade (35 genes), phosphate metabolic process and phosphorus metabolic process (29 genes), protein localization (28 genes), protein transport and establishment of protein localization (26 genes), phosphorylation (25 genes), protein amino acid phosphorylation (23 genes), sensory perception of light stimulus and visual perception (17 genes), small GTPase mediated signal transduction (15 genes), macromolecule catabolic process (14 genes), response to radiation and enzyme linked receptor protein signaling pathway (11 genes), and response to light stimulus (9 genes). However, cell adhesion and biological adhesion (18 genes), macromolecule catabolic process (14 genes), cellular macromolecule catabolic process (13 genes), intracellular transport (13 genes), and membrane organization (10 genes) were enriched with $p$ value $\leq 0.05$ (Figure 1, Additional File S1, Sheet 1, in Supplementary Material available online at http://dx.doi.org/10.1155/2015/638679). The enrichment of genes in GO terms, namely, sensory perception of light stimulus, visual perception, response to light stimulus, response to radiation, cell adhesion, and biological adhesion, was consistent with retinal transcriptome of aged human and rat $[25,26]$.

3.3.2. GO: Cellular Component. The enrichment of expressed genes in cellular component was observed for 293 genes which ranged between 102 and 5 genes in 34 GO terms ( $p$ value $<0.01$ ), namely, plasma membrane part (65 genes), endoplasmic reticulum (37 genes), Golgi apparatus (33 genes), organelle membrane (28 genes), vesicle (25 genes), cytoplasmic vesicle (24 genes), membrane-bounded vesicle (22 genes), cell projection (21 genes), cytoplasmic membranebounded vesicle and endomembrane system (21 genes), vacuole and internal side of plasma membrane (17 genes), lysosome, lytic vacuole (15 genes), proteinaceous extracellular matrix (16 genes), extracellular matrix (16 genes), intracellular organelle lumen (15 genes), and organelle lumen (15 genes). However, GO terms, plasma membrane (102 genes), cytosol (14 genes), anchored to membrane (12 genes), and perinuclear region of cytoplasm (10 genes), were enriched with $p$ value $\leq 0.05$ (Figure 2, Additional File S1, Sheet 2 ). The cellular component GO terms assigned to retinal transcriptome of aged human and mice also showed the GO terms like plasma membrane in human [25] and rat [26].

3.3.3. GO: Molecular Function. The enrichment of expressed genes in cellular component was observed for 293 genes which ranged between 75 and 6 genes in 27 GO terms, namely, nucleotide binding (75 genes), purine nucleotide binding (73 genes), purine ribonucleotide binding, ribonucleotide binding (70 genes), purine nucleoside binding (51 genes), nucleoside binding (51 genes), adenyl nucleotide 


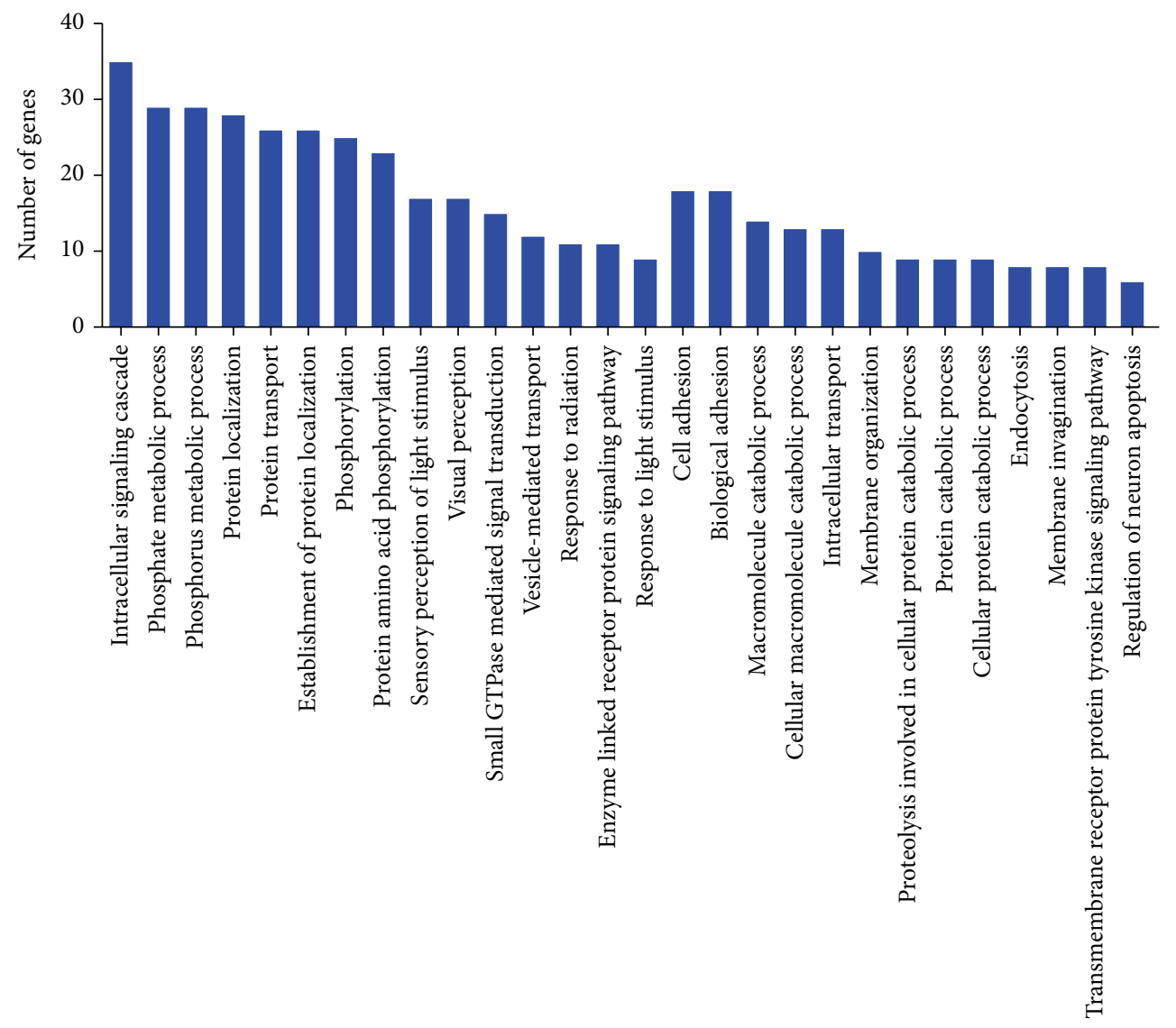

FIGURE 1: Functional annotation of expressed genes of retinal tissue in GO term: biological process.

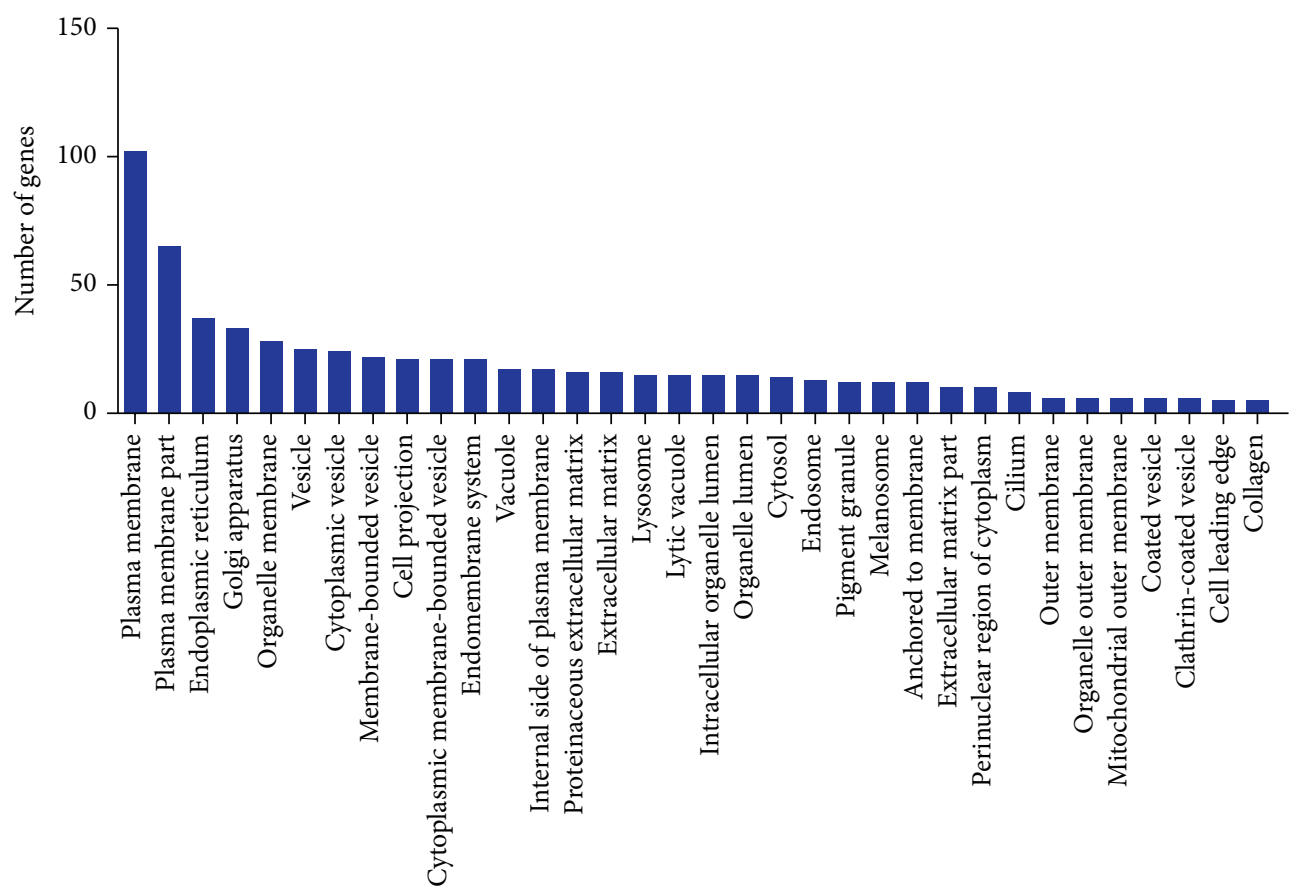

FIGURE 2: Functional annotation of expressed genes of retinal tissue in GO term: cellular component. 
TABLE 2: Distribution of mapped reads to different transcript types and gene regions with reference Canis lupus familiaris genome build version CanFam3.1.

\begin{tabular}{|c|c|c|c|c|}
\hline & \multirow{2}{*}{$\begin{array}{l}\text { Uniquely mapped } \\
\text { Number of reads }\end{array}$} & \multirow{2}{*}{$\begin{array}{c}\text { Nonspecifically mapped } \\
\text { Number of reads }\end{array}$} & \multicolumn{2}{|c|}{ Mapped reads } \\
\hline & & & Number of reads & $\%$ \\
\hline Total exon reads & 75,883 & 1,305 & 77,188 & 34.05 \\
\hline Exon-exon reads & 32,385 & 768 & 33,153 & 14.63 \\
\hline Exon-intron reads & 6,210 & 35 & 6,245 & 2.75 \\
\hline Total intron reads & 107,818 & 2,280 & 110,098 & 48.57 \\
\hline Total gene reads & 222,296 & 4,388 & 226,684 & 100.00 \\
\hline
\end{tabular}

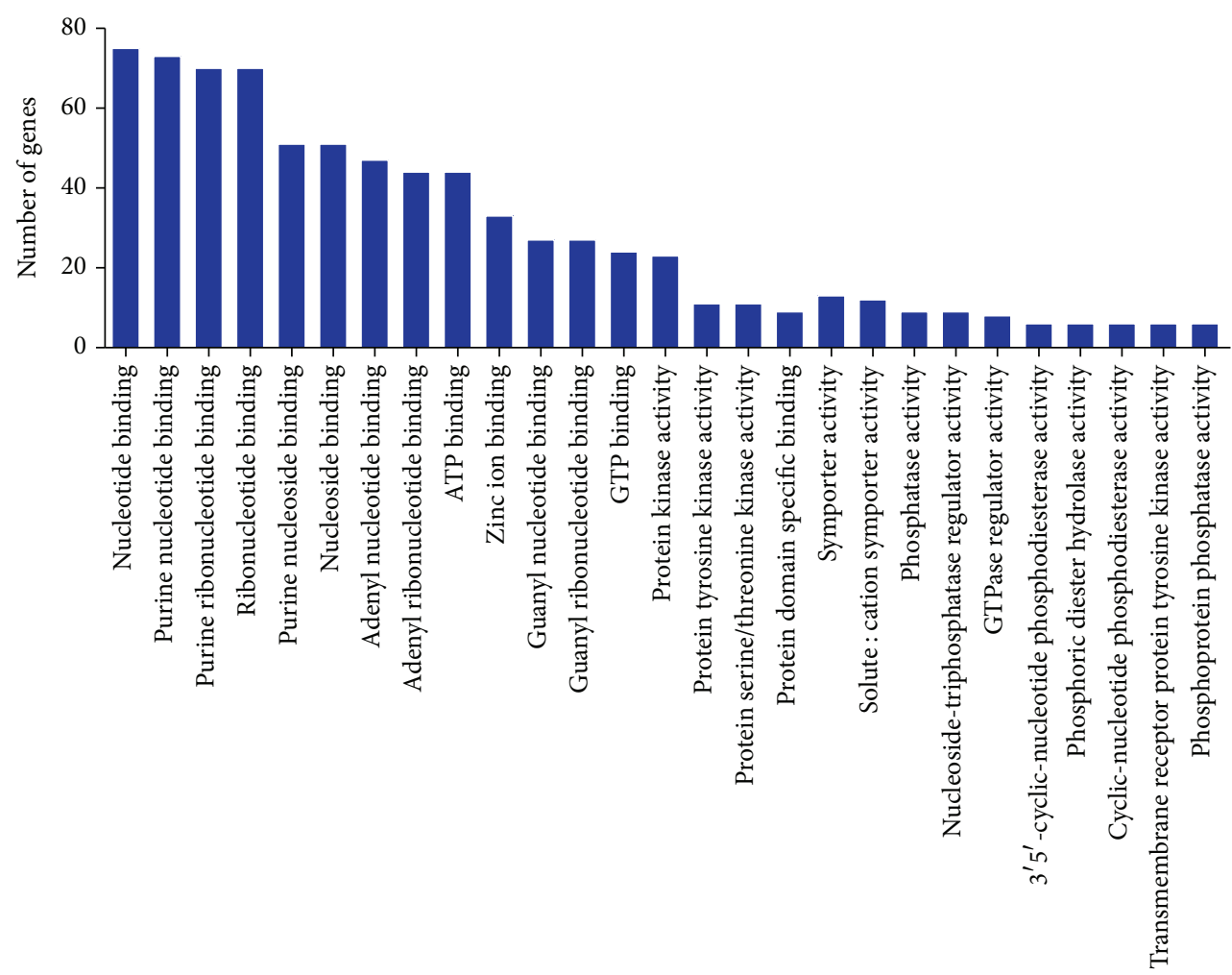

FIGURE 3: Functional annotation of expressed genes of retinal tissue in GO term: molecular function.

binding (47 genes), adenyl ribonucleotide binding (44 genes), ATP binding (44 genes), zinc ion binding (33 genes), guanyl nucleotide binding (27 genes), guanyl ribonucleotide binding (27 genes), GTP binding ( 24 genes), protein kinase activity (23 genes), protein tyrosine kinase activity (11 genes), protein serine/threonine kinase activity (11 genes), and protein domain specific binding (9 genes). In addition, symporter activity (13 genes), solute: cation symporter activity (12 genes), phosphatase activity (9 genes), and nucleosidetriphosphatase regulator activity (9 genes) were enriched with $p$ value $\leq 0.01$ (Figure 3, Additional File S1, Sheet 3 ). This is consistent with retinal transcriptome reports on aged human [25] and rat [26].

3.3.4. Retina-Specific Gene Expression Profiles. In an attempt to determine the retina associated expression of candidate genes, expression of 160 genes out of 221 genes (based on RetNet (https://sph.uth.edu/retnet/home.htm) gene list, January 2014) was detected. Among that, candidate genes like RHO, PDC, RPGR, CNGB1, SLC1A2, SLC1A3, SLC24A1, PRCD, PDE6G, PDE6A, PDE6B, PDE6H, and PDE6C were detected (expression values were presented in Additional File S1, Sheet 4). The GO analysis revealed that all of these genes were enriched in the categories of sensory perception of light stimulus, visual perception, response to radiation, and response to light stimulus (Table 3).

3.4. Assembly of Transcriptome and Comparative Analysis. The reads were assembled using CAP3 which generated a total of 29,683 contigs with N50 of 619 and mean length of 560.9 bases (Table 4). Most of the contigs were in the range between $400 \mathrm{bp}$ and $500 \mathrm{bp}$ (Figure 4). The assembled sequences were compared against the NCBI nr database (ftp://ftp.ncbi.nlm.nih.gov/blast/db) using BLASTx ( $e$-value $\left.1 * 10^{-6}\right)$. Of the 29,683 assembled sequences, $12,498(42.10 \%)$ contigs had significant hits corresponding to a single or more than one unique accession number to the nr database. The 
TABLE 3: GO terms biological process enrichment of expressed genes in retinal tissue.

\begin{tabular}{|c|c|c|c|c|}
\hline GO ID & GO terms & Count & $p$ value & Genes \\
\hline GO:0050953 & $\begin{array}{l}\text { Sensory perception of } \\
\text { light stimulus }\end{array}$ & 17 & 0.006 & $\begin{array}{l}\text { GNAT1, RP1, RPGR, RPE65, RCVRN, PRPH2, PDE6G, CNGA1, GUCY2D, } \\
\text { SAG, PDE6A, PDE6B, PDE6D, PRCD, PDC, CLN5, and RHO }\end{array}$ \\
\hline GO:0007601 & Visual perception & 17 & 0.006 & $\begin{array}{l}\text { GNAT1, RP1, RPGR, RPE65, RCVRN, PRPH2, PDE6G, CNGA1, GUCY2D, } \\
\text { SAG, PDE6A, PDE6B, PDE6D, PRCD, PDC, CLN5, and RHO }\end{array}$ \\
\hline GO:0009314 & Response to radiation & 11 & 0.011 & $\begin{array}{l}\text { GNAT1, GNGT1, SLC1A2, SLC1A3, UACA, CASP9, BAX, RCVRN, } \\
\text { BCL2L1, SNAI2, and RHO }\end{array}$ \\
\hline GO:0009416 & $\begin{array}{l}\text { Response to light } \\
\text { stimulus }\end{array}$ & 9 & 0.017 & $\begin{array}{c}\text { GNAT1, GNGT1, SLC1A2, SLC1A3, UACA, CASP9, BAX, RCVRN, and } \\
\text { RHO }\end{array}$ \\
\hline
\end{tabular}

TABle 4: Summary statistics of C. lupus familiaris reads and assembled contigs.

\begin{tabular}{lc}
\hline Features & Values \\
\hline Clean reads for assembly & 800,154 \\
N50 length & 619 \\
N75 length & 485 \\
N90 length & 384 \\
Contig number & 29,683 \\
Contig bases & $16,649,067$ \\
Maximum contig length & 6,416 \\
Minimum contig length & 42 \\
Mean length of cleaned reads & 560.9 \\
Mode length of cleaned reads & 510 \\
Median length of cleaned reads & 501 \\
Number of reads per contig & 27.95 \\
\hline
\end{tabular}

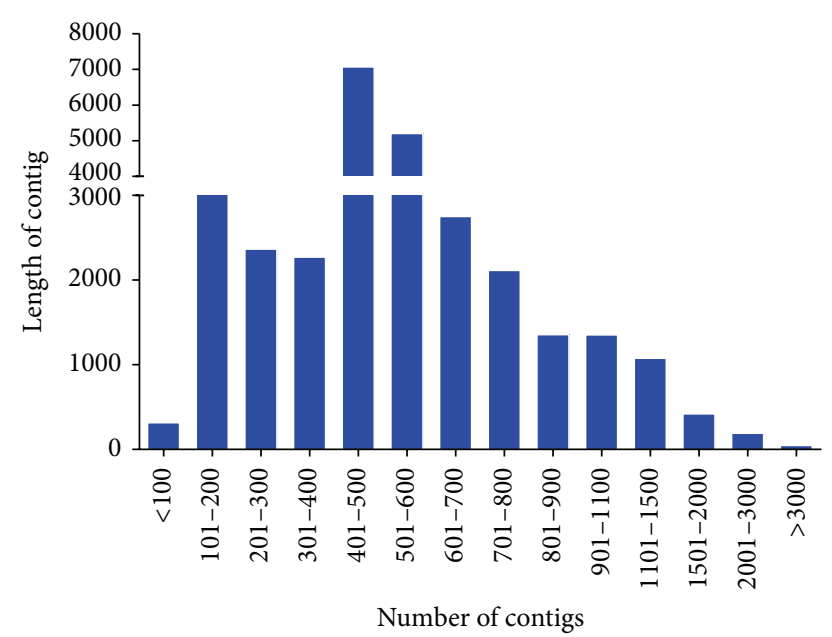

Figure 4: Distribution of contigs length in canine transcriptome assembly.

sequences hits with nr database using BLASTx were $42.10 \%$ with known functions.

3.5. Full-Length cDNA Prediction. Full-length cDNAs are important resources for many genetic and genomic researchers and useful to predict protein sequences [27]. All contigs were analysed by online tool Target Identifier to identify potential full-length cDNAs with complete open reading frame $(\mathrm{ORF})$ in assembled transcriptome of canine retina. A total of 3,827 full-length, 914 short full-length, 331 ambiguous, 2,932 partial ( $5^{\prime}$-sequenced partial), and 2,913 $3^{\prime}$-sequenced partial sequences were identified with a cutoff $e$-value of $10^{-5}$ (Figure 6).

3.6. Open Reading Frame (ORF) Identification. Open reading frame identification in RNA-Seq is important in gene prediction and identification of candidate protein coding regions [27]. In the present study, out of 29,683 total assembled contigs of canine retina transcriptome, 29,418 (99\%) open reading frames (ORFs) were identified with an average length of $285 \mathrm{bp}$ ranging from $50 \mathrm{bp}$ to $5,696 \mathrm{bp}$ (Figure 7). The remaining 265 contigs contained no ORFs, which indicates that these contigs were noncoding or originated from untranslated regions (UTRs).

3.7. Analysis of SSR/Microsatellites. Out of 29,683 contigs, a total of 2,470 SSRs/microsatellites were identified in 2,298 contigs, including dinucleotide $(1,582)(68.84 \%)$, trinucleotide (592) (25.76\%), tetranucleotide (216) (9.40\%), pentanucleotide (58) (2.52\%), and hexanucleotide (22) $(0.96 \%)$ (Figure 8$)$. The most predominant types among the dinucleotide repeat motifs were $(\mathrm{AG} / \mathrm{GA})_{n},(\mathrm{CT} / \mathrm{TC})_{n}$, $(\mathrm{AC} / \mathrm{CA})_{n},(\mathrm{GT} / \mathrm{TG})_{n},(\mathrm{AT} / \mathrm{TA})_{n}$, and $(\mathrm{CG} / \mathrm{GC})_{n}$, with frequencies (in percentage) of 27.88, 23.45, 18.14, 16.56, 11.95 , and 1.71 , respectively. In the 20 types of trinucleotide repeats, GGC (15.20\%) was the major common motif, followed by GCC (12.16\%), TCC (9.46\%), GGA (8.78\%), GTC $(6.42 \%)$, and TTC (5.91\%) repeat motifs (Figure 8 ). These SSRs markers offer a valuable resource for further genetic investigations.

3.8. KEGG Pathway Annotation. In order to identify the active biological pathways in canine tissue, the assembled contigs were used to obtain the enzyme commission (EC) against the Kyoto Encyclopaedia of Genes and Genomes (KEGG) database. A total of 3,782 contigs were assigned to 3,570 enzyme commission (EC) numbers (Table 5). The assignments of contigs with metabolism pathways were predominant. Enzymes involved in retinal tissue metabolism were further classified into 12 subcategories. There were considerably a higher number of enzymes participating in the metabolism of carbohydrate (238 pathways), lipid (167 
TABLE 5: KEGG biochemical mappings for C. lupus familiaris: enzyme commission assignment of assembled transcripts.

\begin{tabular}{|c|c|}
\hline KEGG pathway & $\begin{array}{l}\text { EC count } \\
\text { (unique } \\
\text { transcripts) }\end{array}$ \\
\hline Metabolism & 1213 \\
\hline Overview & 135 \\
\hline Carbohydrate metabolism & 238 \\
\hline Energy metabolism & 96 \\
\hline Lipid metabolism & 176 \\
\hline Nucleotide metabolism & 79 \\
\hline Amino acid metabolism & 164 \\
\hline Metabolism of other amino acids & 53 \\
\hline Glycan biosynthesis and metabolism & 115 \\
\hline Metabolism of cofactors and vitamins & 67 \\
\hline Metabolism of terpenoids and polyketides & 19 \\
\hline Biosynthesis of other secondary metabolites & 17 \\
\hline Xenobiotics biodegradation and metabolism & 54 \\
\hline Genetic Information Processing & 204 \\
\hline Transcription & 21 \\
\hline Translation & 54 \\
\hline Folding, sorting, and degradation & 97 \\
\hline Replication and repair & 32 \\
\hline Environmental Information Processing & 340 \\
\hline Signal transduction & 330 \\
\hline Signaling molecules and interaction & 10 \\
\hline Cellular Processes & 276 \\
\hline Transport and catabolism & 115 \\
\hline Cell motility & 18 \\
\hline Cell growth and death & 70 \\
\hline Cell communication & 73 \\
\hline Organismal Systems & 705 \\
\hline Immune system & 151 \\
\hline Endocrine system & 196 \\
\hline Circulatory system & 26 \\
\hline Digestive system & 67 \\
\hline Excretory system & 37 \\
\hline Nervous system & 163 \\
\hline Sensory system & 15 \\
\hline Development & 30 \\
\hline Environmental adaptation & 20 \\
\hline Human Diseases & 832 \\
\hline Cancers & 320 \\
\hline Immune diseases & 23 \\
\hline Neurodegenerative diseases & 114 \\
\hline Substance dependence & 42 \\
\hline Cardiovascular diseases & 12 \\
\hline Endocrine and metabolic diseases & 24 \\
\hline Infectious diseases & 297 \\
\hline Total & 3570 \\
\hline
\end{tabular}

pathways), amino acid (164 pathways), and glycan biosynthesis and metabolism (115 pathways) (Table 5), indicating enormous tissue activities. Assignment to EC number of human disease was 832 (23.31\%), which included cancers, infectious diseases, neurodegenerative disease, and other categories. Organismal system EC number assignment was 705 (19.75\%), which included endocrine system, nervous system, and immune system. Environmental Information Processing enzymes were 340 (9.52\%) which included signal transduction and signalling molecule interaction. Cellular Processes were 276 (7.73\%) which included transport and catabolism, cell communication, cell growth and death, and cell motility. Genetic Information Processing was 204 (5.71\%) which included folding sorting and degradation, translation, replication and repair, and transcription (Table 5).

3.8.1. Possible Genes Related to Melanogenesis, Phototransduction, and Retinol Metabolism. The transcriptome of canine tissue was primarily examined to identify a wide range of candidate genes that might be functionally associated with melanogenesis, phototransduction, and retinol metabolism (Additional File 2, Table S1 a). The present study indicated that 33 contigs were associated with melanogenesis pathways including adenylate cyclase with the EC number EC:4.6.1.1 encoded by five contigs, while protein kinase A (EC:2.7.11.11), CREB-binding protein (EC:2.3.1.48), RAF protooncogene serine/threonine-protein kinase (EC:2.7.11.1), and mitogen-activated protein kinase kinase 1 (EC:2.7.12.2) were encoded by two contigs. However, mitogen-activated protein kinase 1/3 (EC:2.7.12.2), dopachrome tautomerase (EC:5.3.3.12), phosphatidylinositol phospholipase C, beta (EC:3.1.4.11), calcium/calmodulin-dependent protein kinase (CaMkinase) II (EC:2.7.11.17), and classical protein kinase C (EC:2.7.11.13) were encoded by one contig (Additional File 2). Contigs associated with phototransduction pathways were 15 , with rhodopsin kinase (EC:2.7.11.14, 1 contig) and rod cGMPspecific $3^{\prime}, 5^{\prime}$-cyclic phosphodiesterase (EC:3.1.4.35, 2 contigs) (Additional File 2, Table S1 b). Enzymes involved in retinol metabolism were also encoded by the canine retinal tissue contigs (11 contigs). In the retinol metabolism, main enzymes detected were, namely, alcohol dehydrogenase (EC:1.1.1.1, 1 contig), retinol dehydrogenase (EC:1.1.1, 4 contigs), retinoid isomer hydrolase (EC:3.1.1.64, 3 contigs), and retinal dehydrogenase (EC:1.2.1.36, 1 contig) (Additional File 2, Table S1 c).

\section{Discussion}

We performed RNA-Seq of canine retina using 454 GS-FLX and Ion Torrent PGM. Mapping identified the expression of 10,360 genes out of 28,455 reference genes of canine genome. The expressed genes were utilized for the gene ontology analysis and functional annotation.

In the retinal transcriptome, 316 genes were found to be enriched into the 28 molecular function GO category ( $p$ value $<0.01)$. The enrichment of genes in GO terms, namely, sensory perception of light stimulus, visual perception, response to light stimulus, response to radiation, cell adhesion, and biological adhesion, was consistent with retinal transcriptome of aged human and rat $[25,26]$. The enrichment of genes to these functional categories was further established by RetNet (https://sph.uth.edu/retnet/home.htm) database. These visual functions together serve as the series of events 


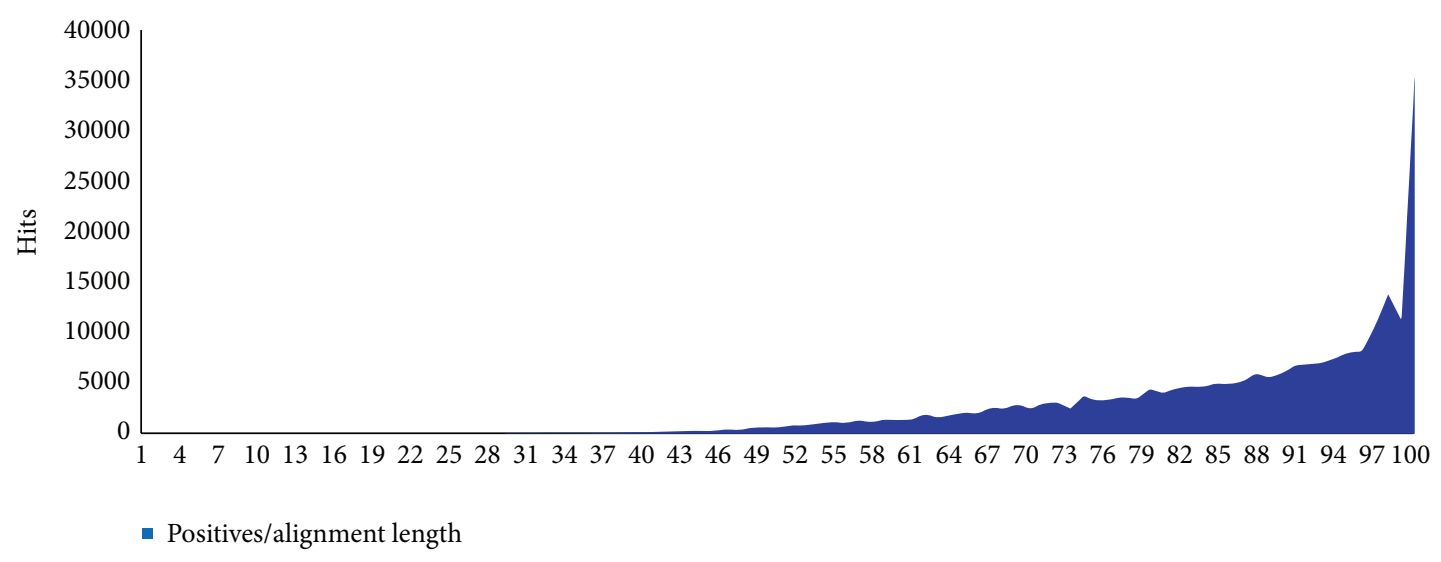

(a)

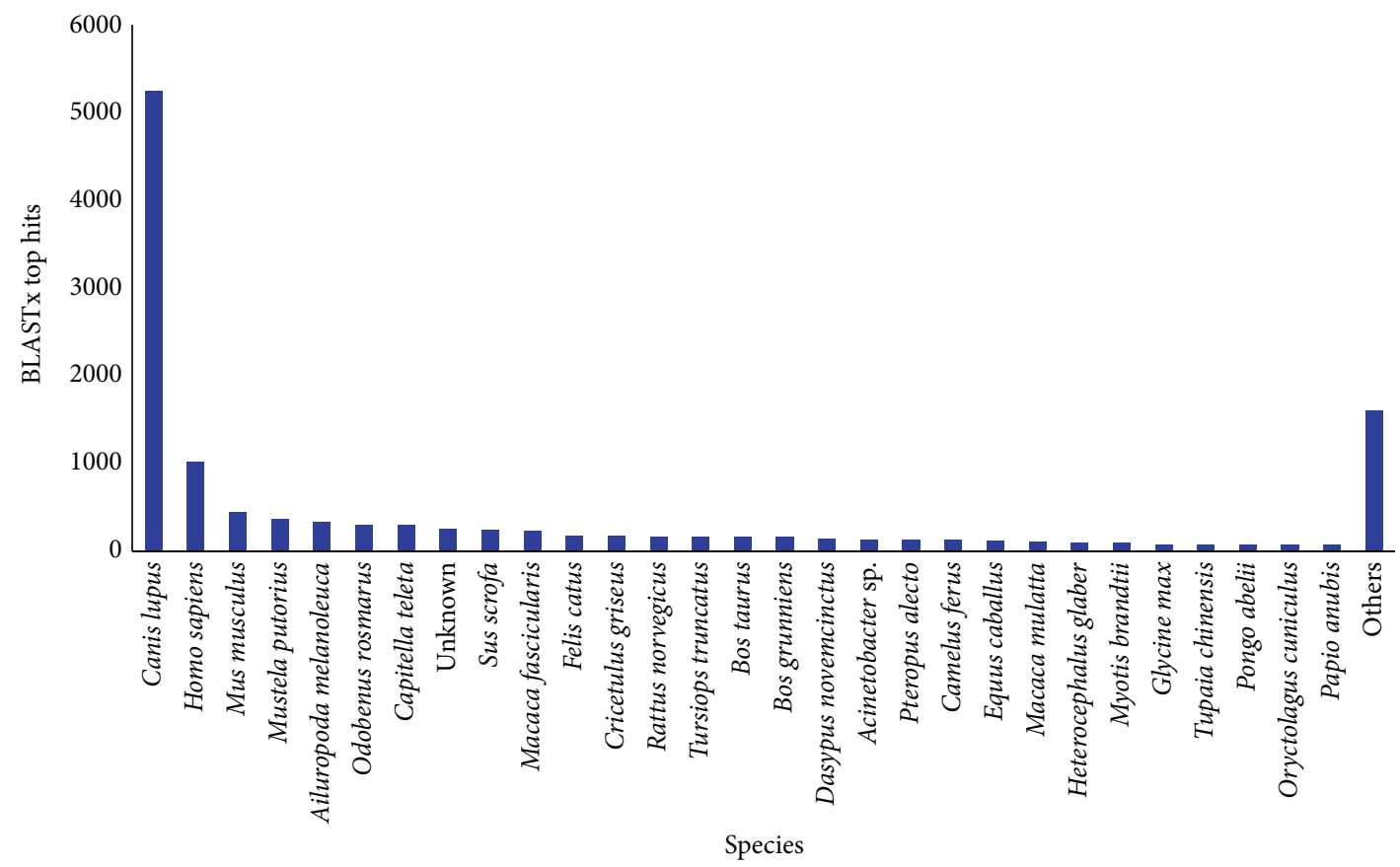

(b)

FIGURE 5: BLASTx hits distribution with $e$-value $10^{-6}$ against NCBI nr database and sequence similarity (a) and species distribution (b).

required for an organism to receive a stimulus, convert it to a molecular signal, and recognize and characterize the signal.

Cellular component refers to the place in the cell where a gene product is active. These terms reflect our understanding of eukaryotic cell structure. In the retinal transcriptome, 293 genes were found to be enriched into the 34 cellular component GO category ( $p$ value $<0.01$ ). The cellular component GO terms assigned to retinal transcriptome of aged human and mice also showed the GO terms like plasma membrane in human [25] and rat [26]. Molecular function refers to the elemental activity or task performed, or potentially performed, by individual gene products. In the retinal transcriptome, 293 genes were found to be enriched into the 27 molecular function GO category ( $p$ value $<0.01$ ).

The BLASTx search of contigs revealed $42 \%$ of the hits matching Canis lupus whereas $8.12 \%$ matched Homo sapiens. Further $e$-value score and top hits distribution shows that maximum sequences were at the range of 90$100 \%$ similarity to reference. However, the poor annotation efficiency could be due to insufficient sequences in public databases for phylogenetically closely related species to date and limited sequence similarity of assembled contigs against NCBI nr database (Figure 5). Additionally, sequences without annotations may represent poorly conserved regions (e.g., untranslated regions (UTRs)) in Canis lupus familiaris. These values were higher than those in the comparable BLAST results from most other published studies using shotgun generated de novo transcriptomes [28-30].

Full-length cDNAs are the valuable source of information for many genetic and genomic researchers and can be useful to predict the protein sequences [27]. A total of 3,827 fulllength cDNA sequences were identified which will serve as base for further cloning and gene expression analysis. A total of 29,418 (99\%) ORFs were predicted from 29,683 contigs. 


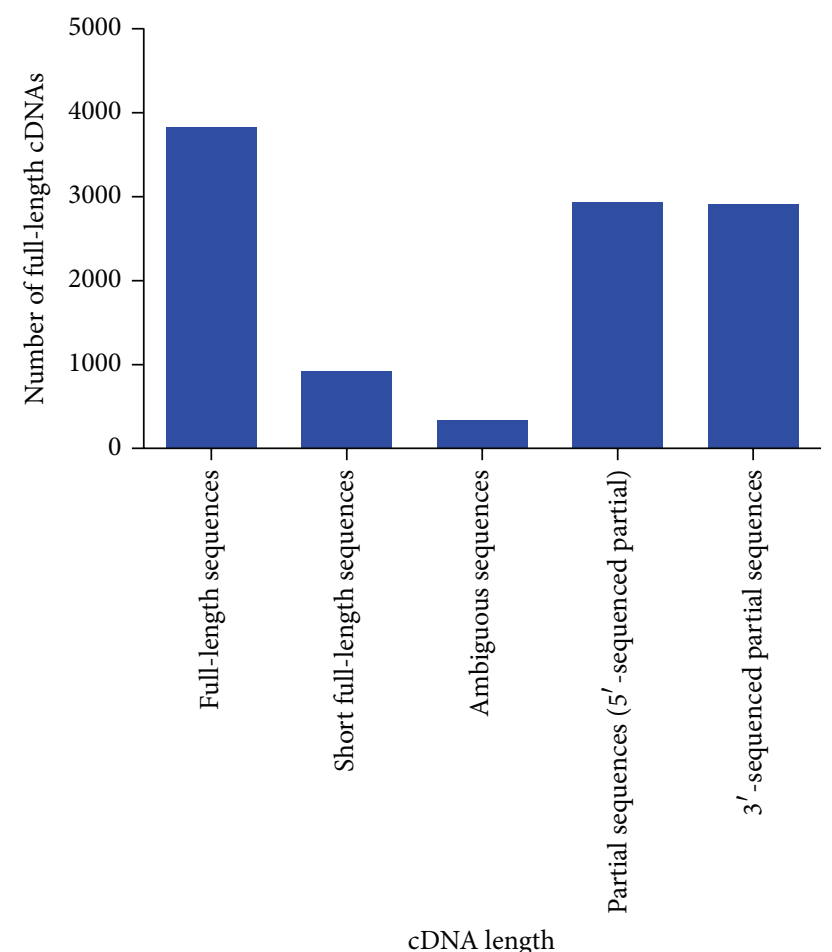

FIGURE 6: Length distribution of putative predicted full-length cDNA of assembled contigs of C. lupus familiaris.

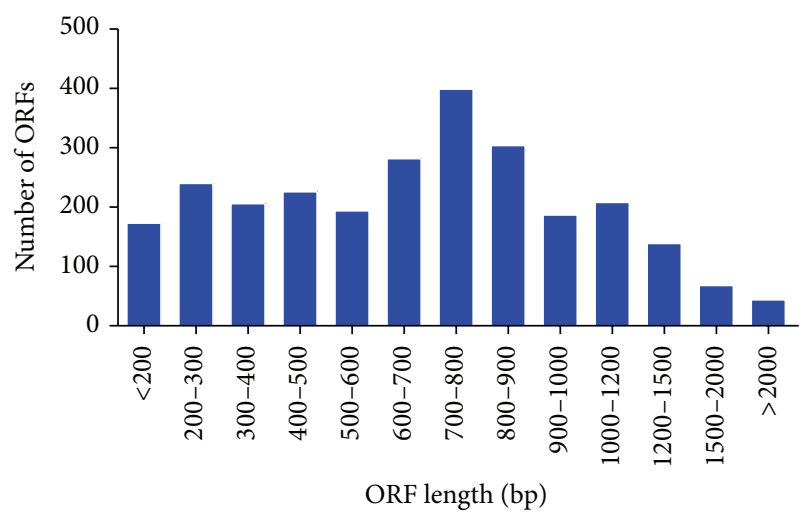

FIGURE 7: Open reading frame (ORF) length distribution of assembled contigs of $C$. lupus familiaris.

These predicted ORFs indicate that most of the contigs have a protein coding sequence and derived from the exonic region of genes. The average length of predicted ORFs was $285 \mathrm{bp}$. The assembled transcriptome contigs can serve as a reference for cSNPs (Coding SNP) identification from transcriptome data for multiple canine breeds. ORF analysis would enable us to discriminate synonymous and nonsynonymous SNPs and to identify nonsense mutations in canines. Next-generation sequencing has identified ORF in Anopheles funestus [31] and plant species [32]. However, there are no reports of predicted ORFs identification in canine to date.
The transcriptome sequencing provides an excellent source for mining and development of gene-associated markers $[33,34]$. Microsatellites or SSRs are molecular markers that are widely distributed in a genome. They consist of repeated core sequences of 2-6 base pairs in length. SSRs have proven to be an efficient tool for performing QTL analysis, constructing genetic linkage, and evaluating the level of genetic variation in a species on account of high diversity, abundance, neutrality, and codominance of microsatellite markers $[35,36]$. In the identified SSRs, the most dominant SSRs were dinucleotide repeats [(AG/GA $)_{n},(\mathrm{CT} / \mathrm{TC})_{n}$, $(\mathrm{AC} / \mathrm{CA})_{n},(\mathrm{GT} / \mathrm{TG})_{n},(\mathrm{AT} / \mathrm{TA})_{n}$, and $\left.(\mathrm{CG} / \mathrm{GC})_{n}\right]$ followed by trinucleotides [GGC, GCC (12.16\%), TCC $(9.46 \%)$, GGA $(8.78 \%)$, GTC (6.42\%), and TTC] (Figure 8). Unlike dog retina, the dinucleotide repeat of AC/GT type is the most abundant in Liaoning cashmere goat [37] compared to other vertebrates [38] but is different from plants [39]. Shotgun sequencing has identified numerous SSRs in plant species [40]. However, there are no reports of SSRs in dog retinal sample in India. We excluded mononucleotide SSRs in our analysis because of the common homopolymer errors that can occur in 454 GS-FLX and Ion Torrent sequencing data. These SSRs markers may offer a valuable resource for genetic variation study and further genetic investigations to be required in large dataset.

Vision is one of the most fascinating mechanisms of the interactions of a biological system and the process of phototransduction where the electromagnetic radiation is converted into biologically recognizable signals by the retinal photoreceptor cell. The phototransduction cascade of vertebrate serves as a benchmark system in signal transduction for a number of light stimuli including the remarkable ability of rod cells to respond reliably to single photon $[41,42]$. In this study, we detected numerous contigs encoding EC number to phototransduction pathway. Strunnikova et al. [27] noted that several of the highly expressed signature genes encode proteins involved in visual cycle, melanogenesis, and cell adhesion in retinal pigment epithelium. The melanogenesis is essential for removal of toxic substances from the choroid and protects the retina from oxidative and chemical stress $[43,44]$. The retinol metabolism (biosynthesis of Vitamin A) is essential for the life of all chordates. It has numerous important functions including a role in vision, maintenance of epithelial surfaces, and immune competence [27]. Multiple genes have been characterized to encode the components of this cycle and linked to many human retinal diseases [45].

\section{Conclusion}

In the present study, we have analyzed transcriptome data and identified $>10000$ expressed genes in canine retinal tissue. The enrichment of genes in GO terms, namely, sensory perception of light stimulus, visual perception, response to radiation, and response to light stimulus, suggested the abundance of genes specifically present in retinal tissue and involved in vision related processes. Several highly expressed genes encoding proteins involved in melanogenesis, phototransduction, and retinol metabolism were identified in the retina. Moreover, a large number of cDNA SSRs were predicted which can be 


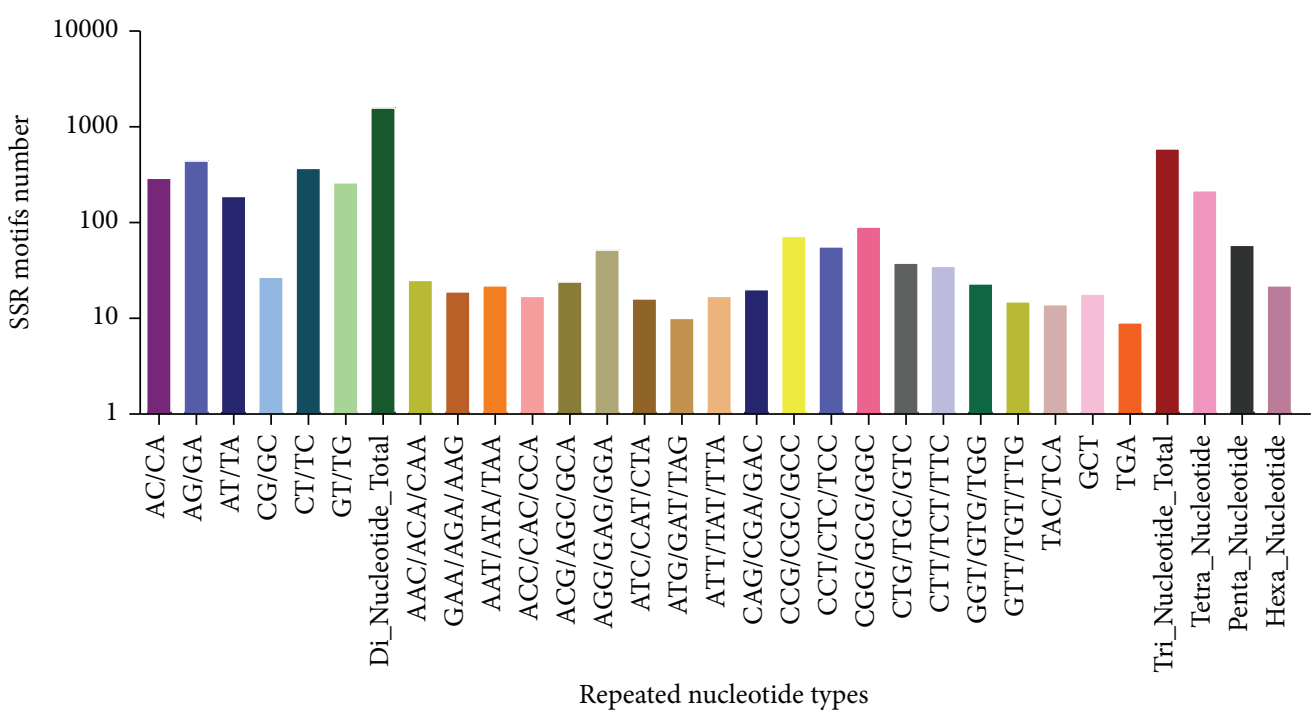

FIGURE 8: Distribution of simple sequence repeats (SSRs) among different nucleotide types found in the transcriptome of $C$. lupus familiaris assembled sequence.

used for subsequent marker development, genetic linkage, and QTL analysis. Overall, the canine retina transcriptome represents a valuable resource for future functional and comparative genomic studies for effective way to treat vision related problem of this globally vulnerable species.

\section{Abbreviations}

RPE: Retinal pigment epithelium

IRD: Inherited retinal degeneration

RPKM: Reads per kilobase per million mapped reads

EST: $\quad$ Expressed Sequence Tag

GO: Gene ontology

CAP3: Contig assembly program-3

ORF: Open reading frame

KEGG: Kyoto Encyclopedia of Genes and Genomes

nr: Nonredundant

SSR: Simple sequence repeat.

\section{Conflict of Interests}

The authors have no conflict of interests in this study.

\section{Acknowledgments}

The present study was carried out by financial support provided by the Department of Biotechnology, Government of India, New Delhi. The authors wish to thank Dr. J. V. Solanki, Scientist Emeritus, College of Veterinary Science and Animal Husbandry, Anand, for critical reading of the paper.

\section{References}

[1] J. E. Dowling, The Retina: An Approachable Part of the Brain, Harvard University Press, Cambridge, Mass, USA, 2012.
[2] D.-G. Luo, T. Xue, and K.-W. Yau, "How vision begins: an odyssey," Proceedings of the National Academy of Sciences of the United States of America, vol. 105, no. 29, pp. 9855-9862, 2008.

[3] O. Strauss, "The retinal pigment epithelium in visual function," Physiological Reviews, vol. 85, no. 3, pp. 845-881, 2005.

[4] S. Khandhadia, J. Cherry, and A. J. Lotery, "Age-related macular degeneration," Advances in Experimental Medicine and Biology, vol. 724, pp. 15-36, 2012.

[5] L. R. Pacione, M. J. Szego, S. Ikeda, P. M. Nishina, and R. R. McInnes, "Progress toward understanding the genetic and biochemical mechanisms of inherited photoreceptor degenerations," Annual Review of Neuroscience, vol. 26, pp. 657-700, 2003.

[6] J. K. Phelan and D. Bok, "A brief review of retinitis pigmentosa and the identified retinitis pigmentosa genes," Molecular Vision, vol. 6, no. 16, pp. 116-124, 2000.

[7] A. Rattner, H. Sun, and J. Nathans, "Molecular genetics of human retinal disease," Annual Review of Genetics, vol. 33, pp. 89-131, 1999.

[8] A. K. Hennig, G.-H. Peng, and S. Chen, "Regulation of photoreceptor gene expression by Crx-associated transcription factor network," Brain Research, vol. 1192, pp. 114-133, 2008.

[9] H. R. Coleman, C.-C. Chan, F. L. Ferris III, and E. Y. Chew, "Agerelated macular degeneration," The Lancet, vol.372, no. 9652, pp. 1835-1845, 2008.

[10] A. Swaroop and D. J. Zack, "Transcriptome analysis of the retina," Genome Biology, vol. 3, no. 8, article 1022, 2002.

[11] S. Blackshaw, R. E. Fraioli, T. Furukawa, and C. L. Cepko, "Comprehensive analysis of photoreceptor gene expression and the identification of candidate retinal disease genes," Cell, vol. 107, no. 5, pp. 579-589, 2001.

[12] C. Trapnell, B. A. Williams, G. Pertea et al., "Transcript assembly and quantification by RNA-Seq reveals unannotated transcripts and isoform switching during cell differentiation," Nature Biotechnology, vol. 28, no. 5, pp. 511-515, 2010.

[13] A. Conesa, S. Götz, J. M. García-Gómez, J. Terol, M. Talón, and M. Robles, "Blast2GO: a universal tool for annotation, 
visualization and analysis in functional genomics research," Bioinformatics, vol. 21, no. 18, pp. 3674-3676, 2005.

[14] F. Ozsolak and P. M. Milos, "RNA sequencing: advances, challenges and opportunities," Nature Reviews Genetics, vol. 12, no. 2, pp. 87-98, 2011.

[15] J. Briggs, M. Paoloni, Q.-R. Chen, X. Wen, J. Khan, and C. Khanna, "A compendium of canine normal tissue gene expression," PLoS ONE, vol. 6, no. 5, Article ID e17107, 2011.

[16] R. Schmieder and R. Edwards, "Quality control and preprocessing of metagenomic datasets," Bioinformatics, vol. 27, no. 6, pp. 863-864, 2011.

[17] A. Cánovas, G. Rincon, A. Islas-Trejo, S. Wickramasinghe, and J. F. Medrano, "SNP discovery in the bovine milk transcriptome using RNA-Seq technology," Mammalian Genome, vol. 21, no. 11-12, pp. 592-598, 2010.

[18] D. W. Huang, B. T. Sherman, and R. A. Lempicki, "Systematic and integrative analysis of large gene lists using DAVID bioinformatics resources," Nature Protocols, vol. 4, no. 1, pp. 44-57, 2009.

[19] X. Huang and A. Madan, "CAP3: a DNA sequence assembly program," Genome Research, vol. 9, no. 9, pp. 868-877, 1999.

[20] Y. Moriya, M. Itoh, S. Okuda, A. C. Yoshizawa, and M. Kanehisa, "KAAS: an automatic genome annotation and pathway reconstruction server," Nucleic Acids Research, vol. 35, no. 2, pp. W182-W185, 2007.

[21] X. J. Min, G. Butler, R. Storms, and A. Tsang, "TargetIdentifier: a webserver for identifying full-length cDNAs from EST sequences," Nucleic Acids Research, vol. 33, supplement 2, pp. W669-W672, 2005.

[22] X. J. Min, G. Butler, R. Storms, and A. Tsang, "OrfPredictor: predicting protein-coding regions in EST-derived sequences," Nucleic Acids Research, vol. 33, no. 2, pp. W677-W680, 2005.

[23] L. C. da Maia, D. A. Palmieri, V. Q. de Souza, M. M. Kopp, F. I. F. de Carvalho, and A. Costa de Oliveira, "SSR locator: tool for simple sequence repeat discovery integrated with primer design and PCR simulation," International Journal of Plant Genomics, vol. 2008, Article ID 412696, 9 pages, 2008.

[24] M. A. Tariq, H. J. Kim, O. Jejelowo, and N. Pourmand, "Wholetranscriptome RNAseq analysis from minute amount of total RNA," Nucleic Acids Research, vol. 39, no. 18, article e120, 2011.

[25] M.-N. Delyfer, W. Raffelsberger, D. Mercier et al., "Transcriptomic analysis of human retinal detachment reveals both inflammatory response and photoreceptor death," PLOS ONE, vol. 6, no. 12, Article ID e28791, 2011.

[26] O. S. Kozhevnikova, E. E. Korbolina, N. I. Ershov, and N. G. Kolosova, "Rat retinal transcriptome: effects of aging and AMDlike retinopathy," Cell Cycle, vol. 12, no. 11, pp. 1745-1761, 2013.

[27] N. V. Strunnikova, A. Maminishkis, J. J. Barb et al., “Transcriptome analysis and molecular signature of human retinal pigment epithelium," Human Molecular Genetics, vol. 19, no. 12, pp. 2468-2486, 2010.

[28] B. Ewen-Campen, N. Shaner, K. A. Panfilio, Y. Suzuki, S. Roth, and C. G. Extavour, "The maternal and early embryonic transcriptome of the milkweed bug Oncopeltus fasciatus," BMC Genomics, vol. 12, article 61, 2011.

[29] E. Meyer, G. V. Aglyamova, S. Wang et al., "Sequencing and de novo analysis of a coral larval transcriptome using 454 GSFlx," BMC Genomics, vol. 10, article 219, 2009.

[30] V. Zeng, K. E. Villanueva, B. S. Ewen-Campen, F. Alwes, W. E. Browne, and C. G. Extavour, "De novo assembly and characterization of a maternal and developmental transcriptome for the emerging model crustacean Parhyale hawaiensis," BMC Genomics, vol. 12, article 581, 2011.

[31] J. E. Crawford, W. M. Guelbeogo, A. Sanou et al., "De novo transcriptome sequencing in Anopheles funestus using illumina RNA-seq technology," PLoS ONE, vol. 5, no. 12, Article ID e14202, 2010.

[32] H. van Bakel, J. M. Stout, A. G. Cote et al., "The draft genome and transcriptome of Cannabis sativa," Genome Biology, vol. 12, article R102, 2011.

[33] W. B. Barbazuk, S. J. Emrich, H. D. Chen, L. Li, and P. S. Schnable, "SNP discovery via 454 transcriptome sequencing," The Plant Journal, vol. 51, no. 5, pp. 910-918, 2007.

[34] E. Novaes, D. R. Drost, W. G. Farmerie et al., "High-throughput gene and SNP discovery in Eucalyptus grandis, an uncharacterized genome," BMC Genomics, vol. 9, article 312, 2008.

[35] Q. Kong, C. Xiang, and Z. Yu, "Development of EST-SSRs in Cucumis sativus from sequence database," Molecular Ecology Notes, vol. 6, no. 4, pp. 1234-1236, 2006.

[36] X.-C. Wang, L. Guo, L.-F. Shangguan et al., "Analysis of expressed sequence tags from grapevine flower and fruit and development of simple sequence repeat markers," Molecular Biology Reports, vol. 39, no. 6, pp. 6825-6834, 2012.

[37] H. Liu, T. Wang, J. Wang, F. Quan, and Y. Zhang, "Characterization of Liaoning cashmere goat transcriptome: sequencing, de novo assembly, functional annotation and comparative analysis," PLoS ONE, vol. 8, no. 10, Article ID e77062, 2013.

[38] U. Lagercrantz, H. Ellegren, and L. Andersson, “The abundance of various polymorphic microsatellite motifs differs between plants and vertebrates," Nucleic Acids Research, vol. 21, no. 5, pp. 1111-1115, 1993.

[39] M. He, Y. Wang, W. Hua, Y. Zhang, and Z. Wang, "De novo sequencing of Hypericum perforatum transcriptome to identify potential genes involved in the biosynthesis of active metabolites," PLoS ONE, vol. 7, no. 7, Article ID e42081, 2012.

[40] F. L. Luro, G. Costantino, J. Terol et al., "Transferability of the EST-SSRs developed on Nules clementine (Citrus clementina Hort ex Tan) to other citrus species and their effectiveness for genetic mapping," BMC Genomics, vol. 9, article 287, 2008.

[41] D. A. Baylor, T. D. Lamb, and K.-W. Yau, "Responses of retinal rods to single photons," The Journal of Physiology, vol. 288, pp. 613-634, 1979.

[42] D. A. Baylor, B. J. Nunn, and J. L. Schnapf, “The photocurrent, noise and spectral sensitivity of rods of the monkey Macaca fascicularis," The Journal of Physiology, vol. 357, pp. 575-607, 1984.

[43] S. Peters, T. Lamah, D. Kokkinou, K.-U. Bartz-Schmidt, and U. Schraermeyer, "Melanin protects choroidal blood vessels against light toxicity," Zeitschrift für Naturforschung C, vol. 61, no. 5-6, pp. 427-433, 2006.

[44] T. Sarna, "New trends in photobiology: properties and function of the ocular melanin-a photobiophysical view," Journal of Photochemistry and Photobiology B: Biology, vol. 12, no. 3, pp. 215-258, 1992.

[45] R. Blomhoff and H. K. Blomhoff, "Overview of retinoid metabolism and function," Journal of Neurobiology, vol. 66, no. 7, pp. 606-630, 2006. 

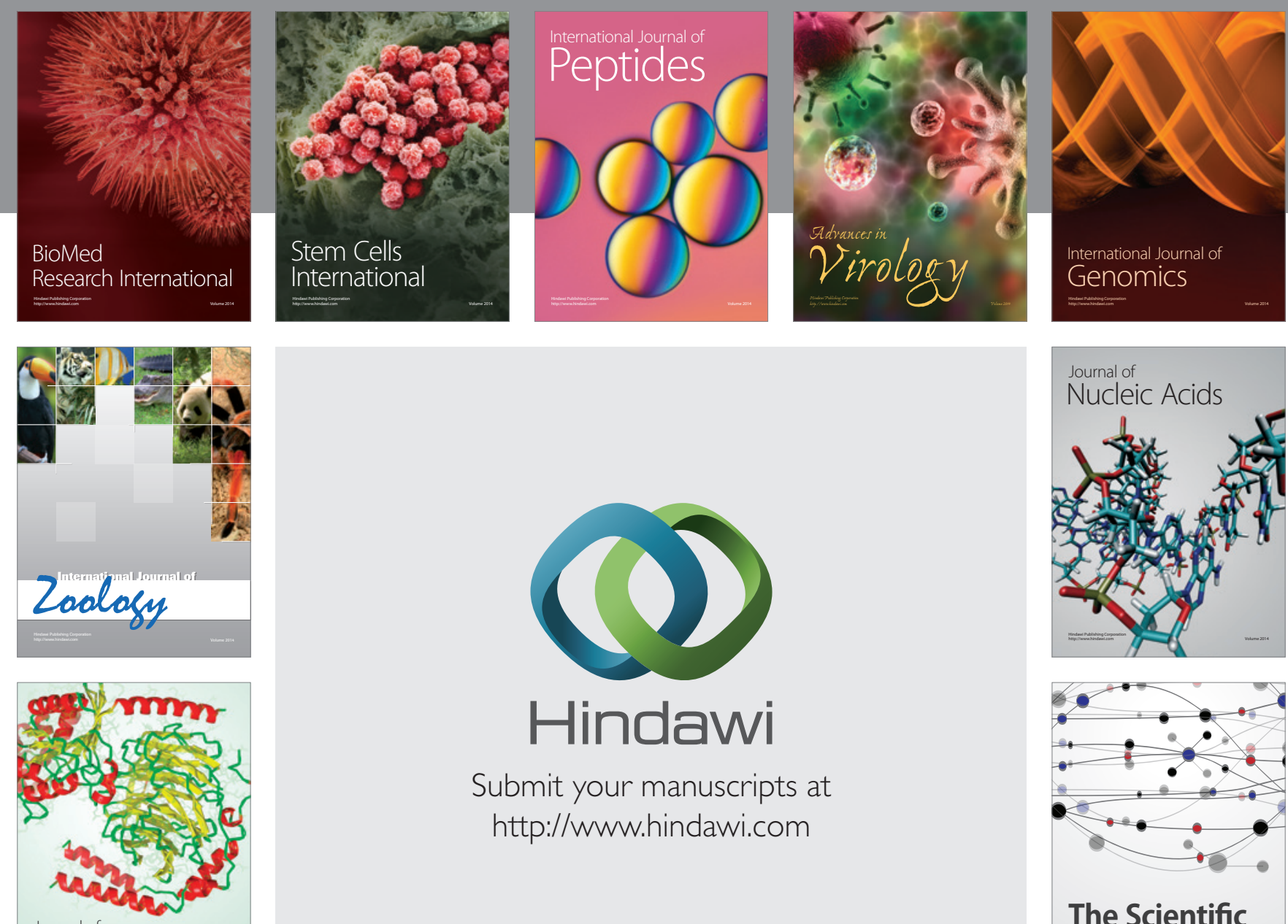

Submit your manuscripts at

http://www.hindawi.com

Journal of
Signal Transduction
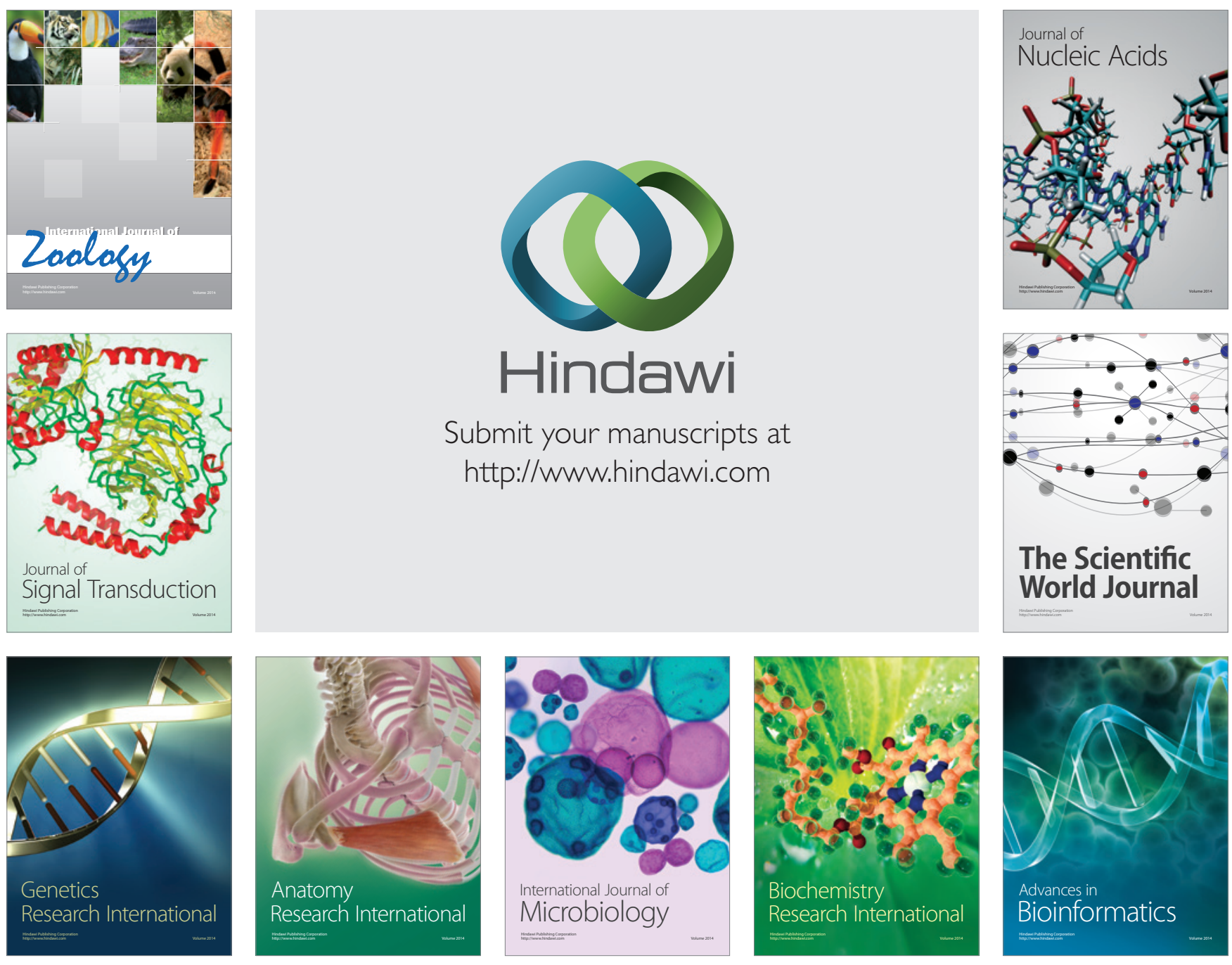

The Scientific World Journal
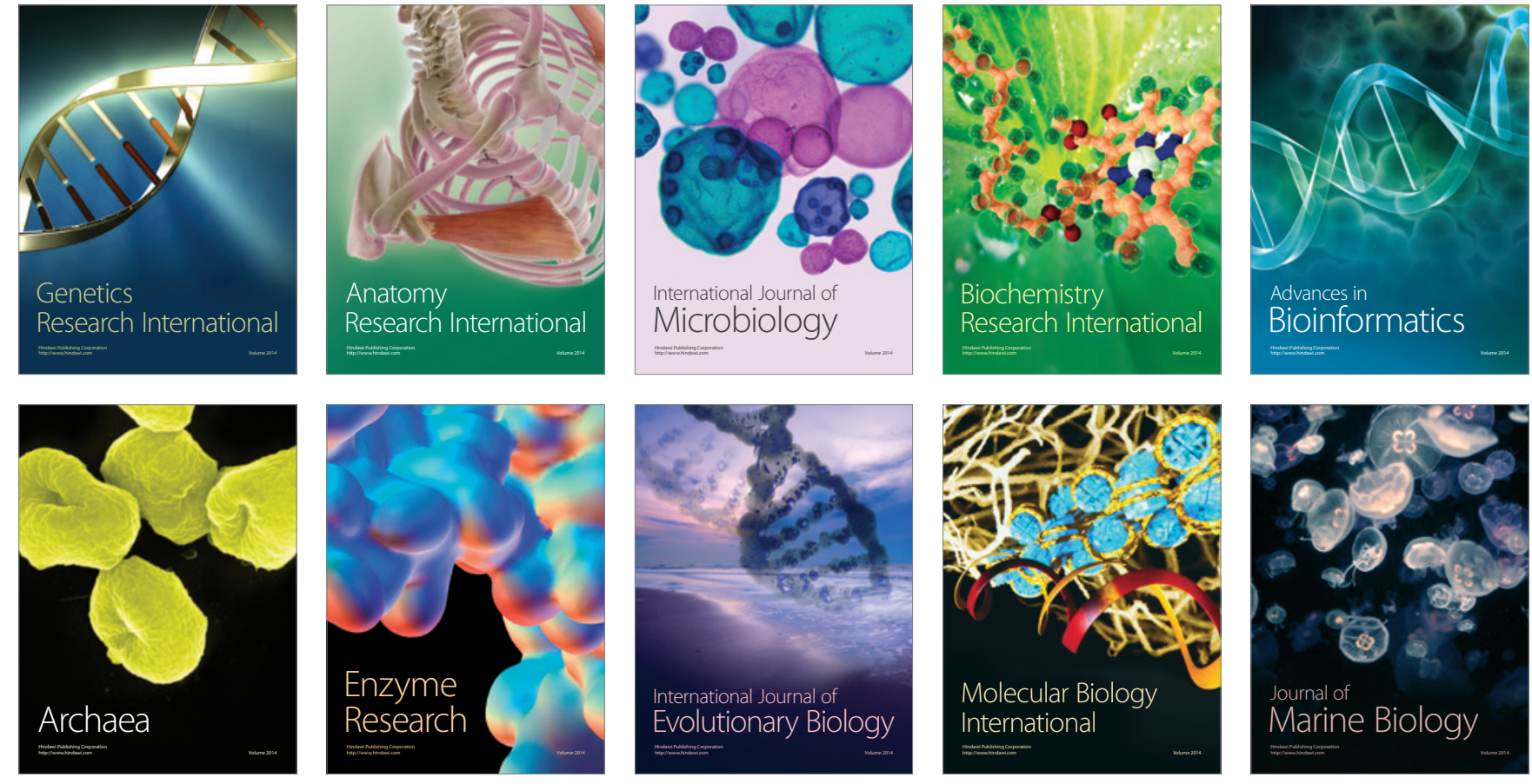